\title{
Inducible and constitutive promoters for genetic systems in Sulfolobus acidocaldarius
}

\author{
Silvia Berkner • Alexander Wlodkowski • \\ Sonja-Verena Albers · Georg Lipps
}

Received: 30 September 2009/Accepted: 5 February 2010/Published online: 12 March 2010

(C) The Author(s) 2010. This article is published with open access at Springerlink.com

\begin{abstract}
Central to genetic work in any organism are the availability of a range of inducible and constitutive promoters. In this work we studied several promoters for use in the hyperthermophilic archaeon Sulfolobus acidocaldarius. The promoters were tested with the aid of an E. coliSulfolobus shuttle vector in reporter gene experiments. As the most suitable inducible promoter a maltose inducible promoter was identified. It comprises $266 \mathrm{bp}$ of the sequence upstream of the gene coding for the maltose/ maltotriose binding protein (mbp, Saci_1165). Induction is feasible with either maltose or dextrin at concentrations of $0.2-0.4 \%$. The highest increase in expression (up to 17-fold) was observed in late exponential and stationary phase around 30-50 $\mathrm{h}$ after addition of dextrin. Whereas in the presence of glucose and xylose higher basal activity
\end{abstract}

Communicated by F. Robb.

\section{S. Berkner}

Department of Biochemistry, University of Bayreuth, Universitätsstr. 30, 95447 Bayreuth, Germany

A. Wlodkowski · S.-V. Albers

Molecular Biology of Archaea,

Max Planck Institute for Terrestrial Microbiology,

Karl-von-Frisch Strasse, 35043 Marburg, Germany

\section{G. Lipps ( $\square)$}

Institute of Chemistry and Bioanalytics, School of Life Sciences, University of Applied Sciences of Northwestern Switzerland (FHNW), Gründenstrasse 40, 4132 Muttenz, Switzerland e-mail: georg.lipps@fhnw.ch

\section{Present Address:}

\section{S. Berkner}

Federal Environment Agency, Section IV 2.2 Pharmaceuticals,

Washing and Cleansing Agents, and Nanotechnology,

Wörlitzer Platz 1, 06844 Dessau, Germany and reduced inducibility with maltose is observed, sucrose can be used in the growth medium additionally without affecting the basal activity or the inducibility. The minimal promoter region necessary could be narrowed down to $169 \mathrm{bp}$ of the upstream sequence. The ABCE1 protein from $S$. solfataricus was successfully expressed under control of the inducible promoter with the shuttle vector $\mathrm{pC}$ and purified from the $S$. acidocaldarius culture with a yield of about $1 \mathrm{mg} \mathrm{L}^{-1}$ culture. In addition we also determined the promoter strength of several constitutive promoters.

Keywords Inducible promoter - Maltose binding protein - Sugar transport operon - Shuttle vector . Reporter gene $\cdot$ Protein expression

\section{Introduction}

Recently, there has been much progress in the field of genetic systems for hyperthermophilic Crenarchaeota (Berkner and Lipps 2008a; Deng et al. 2009; She et al. 2009). For the emerging model organism Sulfolobus acidocaldarius shuttle vectors (Berkner et al. 2007) and gene knockout techniques (Wagner et al. 2009) have been developed. However, an inducible promoter for controlled expression of genes in $S$. acidocaldarius has not been described so far. For $S$. solfataricus the araS promoter is available, which can be induced upon addition of arabinose to the growth medium (Lubelska et al. 2006). The araS promoter has already been used for expression of different proteins in $S$. solfataricus in combination with virus-based shuttle vectors (Albers et al. 2006) and with pRN1-based shuttle vectors (Samson et al. 2008). Unfortunately, $S$. acidocaldarius does not contain an arabinose transport operon like $S$. solfataricus, thus preventing the use of the 
araS promoter in S. acidocaldarius. However, S. acidocaldarius is an important model organism and is more suitable for many molecular biology experiments than S. solfataricus, since among others it is genetically more stable (Berkner and Lipps 2008b) and sophisticated cell cycle synchronization techniques have been developed (Duggin et al. 2008). For that reason, it was crucial to identify and characterize an inducible promoter that can be used in S. acidocaldarius. Constitutive promoters with different strength form another type of promoter that is useful for genetic work, especially for expression purposes.

So far, not many Sulfolobus promoters have been studied in vivo in reporter gene experiments. Besides the already mentioned araS promoter, the $t f 55 \alpha$ promoter from $S$. solfataricus P2 (Sso0282) has been investigated in reporter gene experiments. It has a very high basal activity and is further inducible by heat-shock. In these experiments, cells were grown at $88^{\circ} \mathrm{C}$ for up to $46 \mathrm{~h}$ and a three- to fourfold induction was observed after $46 \mathrm{~h}$ (Jonuscheit et al. 2003). This promoter has also been used in a pRN1-based shuttle vector. However, the retention of this construct (pJlacS) was reduced (Berkner et al. 2007). Furthermore, the heat shock is imposing stress on the cells and is also likely to negatively impact transcription, translation, and replication due to the heat shock response of the cell.

The requirements for an inducible promoter are a low basal activity in combination with a high increase in activity upon induction. The inducer should be easy to handle and stable under the Sulfolobus growth conditions. It should also be considered that the agent used for induction (chemical or physical) should not negatively affect the cells. For protein expression purposes it is not desirable to use an inducer that has secondary effect on the protein synthesis rate. Substances that are also used as nutrients for Sulfolobus, e.g., sugars, belong to a class of inducers that certainly exerts no negative effect on the fitness of the cells. The expression of sugar-binding proteins is most probably regulated in response to the availability of sugars in the growth medium. These binding proteins are part of ATP-binding cassette (ABC) transporters and extracellularly capture sugar molecules and transfer them to the cognate transporter for import into the cytoplasm. In S. solfataricus several different sugar ABCtransport operons have been identified in the genome sequence (Elferink et al. 2001), and the response of the glucose and arabinose transport operons to different sugars and amino acids have been examined (Lubelska et al. 2006). The genes coding for ABC transporter components are frequently organized in operons comprising the genes for the binding protein, permease(s), and ATPase(s). In contrast to $S$. solfataricus, $S$. acidocaldarius contains only very few sugar ABC-transport operons (Albers et al. 2004), which reflects the more restricted substrate spectrum of $S$. acidocaldarius (Grogan 1989).

\section{Materials and methods}

Strains and culture conditions

Sulfolobus acidocaldarius MR31 (Reilly and Grogan 2001) was grown in Brock's basal salt medium at pH 3.5 (Brock et al. 1972). Acid-hydrolyzed casein (NZAmine AS, Sigma, for plates), or enzymatically hydrolyzed casein (tryptone, BD Biosciences, for liquid medium), or a mix of the 20 proteinogenic amino acids (Berkner and Lipps 2008b) was added at $0.1 \%$. Sugars (D- $(+)$-sucrose, D- $(+)$-glucose, $\mathrm{D}-(+)$-xylose, lactose, maltose, and dextrin) were added at 0.2 or $0.4 \%$ as indicated. For growth of untransformed cells or under non-selective conditions, $20 \mu \mathrm{g} \mathrm{mL}^{-1}$ of uracil was added to the medium. Plates were solidified by addition of $0.6 \%$ Gelrite (Sigma) and $10 \mathrm{mM} \mathrm{CaCl}_{2}$. Plates and shake flask cultures were incubated at $75^{\circ} \mathrm{C}$.

\section{Transformation of $S$. acidocaldarius MR31}

Preparation of competent cells, methylation of plasmids, and electroporation was essentially carried out as described in detail in Berkner et al. (2007). The electroporator used was the Gene Pulser Xcell (BioRad) with the following parameters: $1,250 \mathrm{~V}, 25 \mu \mathrm{F}, 1,000 \Omega, 1 \mathrm{~mm}$ cuvettes. Regeneration was done for $30 \mathrm{~min}$ at $75^{\circ} \mathrm{C}$ in recovery solution (Kurosawa and Grogan 2005). After regeneration, cells were plated on NZAmine/sucrose plates and incubated at $75^{\circ} \mathrm{C}$.

Retransformation to check plasmid integrity

Genomic DNA was prepared from $S$. acidocaldarius MR31, retransformed into E. coli, and analyzed as described before (Berkner et al. 2007).

Construction of plasmids

The promoter region of the Sulfolobus solfataricus gene dps (sso2079) was amplified with the primers pCdpsLacS forward and reverse (see Table 1) and cloned upstream of the reporter gene lacS from Sulfolobus solfataricus using the restriction site $B g l l \mathrm{I}$ and $N c o$ I and introducing the restriction site $\mathrm{SacII}$ at the $5^{\prime}$ end of the promoter. The promoter reporter gene fusion was then cloned into the Sulfolobus-E. coli vector pC (Berkner et al. 2007) with aid of the restriction sites SacII and NotI. To construct the remaining promoter reporter gene fusions, promoter regions were amplified by PCR introducing the restriction 
Table 1 List of oligodesoxynucleotides used for amplifying the promoters

\begin{tabular}{|c|c|}
\hline \multicolumn{2}{|c|}{ pCdpsLacS } \\
\hline For & ccgagatctccgcggagacgectcttcaatagttatttccg \\
\hline Rev & ggaaccatggttttctctcccaatatcatact \\
\hline \multicolumn{2}{|c|}{ pCsso_dpsLacS } \\
\hline For & gccgcggttatcttagttaggttttct \\
\hline Rev & gccatggatacaccctaatagtcaata \\
\hline \multicolumn{2}{|c|}{ pCdps_longLacS } \\
\hline For & atcgccgcggtataaggtatgtatacagcattaccgg \\
\hline Rev & atcgccatggttttctctcccaatatcatactagac \\
\hline \multicolumn{2}{|c|}{ pClacSLacS } \\
\hline For & ccgcggetccaccaataaagatcccctg \\
\hline Rev & ccatggacagatataagttgaccaaggc \\
\hline \multicolumn{2}{|c|}{ pCmalLacS } \\
\hline \multirow[t]{4}{*}{ For } & mal gcatccgcggecagatatctgatagttggag \\
\hline & mal2 atcgecgeggtcttttttacctactcettgtgg \\
\hline & mal3 atcgccgcggaatttaagtaaattaaataaaggc \\
\hline & mal4 atcgccgeggcggaggtgtcettaagtttagacc \\
\hline Rev & gcatccatggggttaacttaatcacgtaattatttataaac \\
\hline \multicolumn{2}{|c|}{ pCgdhALacS } \\
\hline For & atcgecgcggttctccactgtttacgtttacttctcc \\
\hline Rev & atcgecatggtttatccaagtaattcttctctctaaac \\
\hline \multicolumn{2}{|c|}{ pCsac7dLacS } \\
\hline For & atcgccgcggecctcactataactagctagtttaagg \\
\hline Rev & atcgccatggtaggtcaagttatcttacgatattgtc \\
\hline
\end{tabular}

The SacII and NcoI restriction sites are underlined. The BglII restriction site used to clone the initial promoter reporter gene fusion is double underlined

sites SacII and NcoI with the primers. The two Cs of the NcoI site always replace the two nucleotides that are present in the native promoter sequence, thus keeping the distance between promoter and start codon the same as in the native sequence. Next, the $d p s$ promoter sequence was swapped using the restriction sites SacII and NcoI. In case of the promoterless construct pCLacS the vector was blunted and religated without insert. A map of the vector pCmalLacS is shown in Fig. 1. Table 2 gives an overview of all different promoter constructs, the names of the genes (and the organism) from which the promoter regions were cloned, and the lengths of the cloned fragments.

The vector for expression of ABCE1 protein (SSO0287) pSVA1401 was constructed by replacing the lacS gene from pCmalLacS with the ABCE1 gene from vector pSVA30 (Albers et al. 2006) by restriction with NcoI/EagI. The ABCE1 protein was thus expressed with a C-terminal tandem tag consisting of a histidine and strep tag. The histidine tag consists of ten consecutive histidine residues and facilitates purification of the protein by metal chelate affinity chromatography. The strep tag encompasses eight

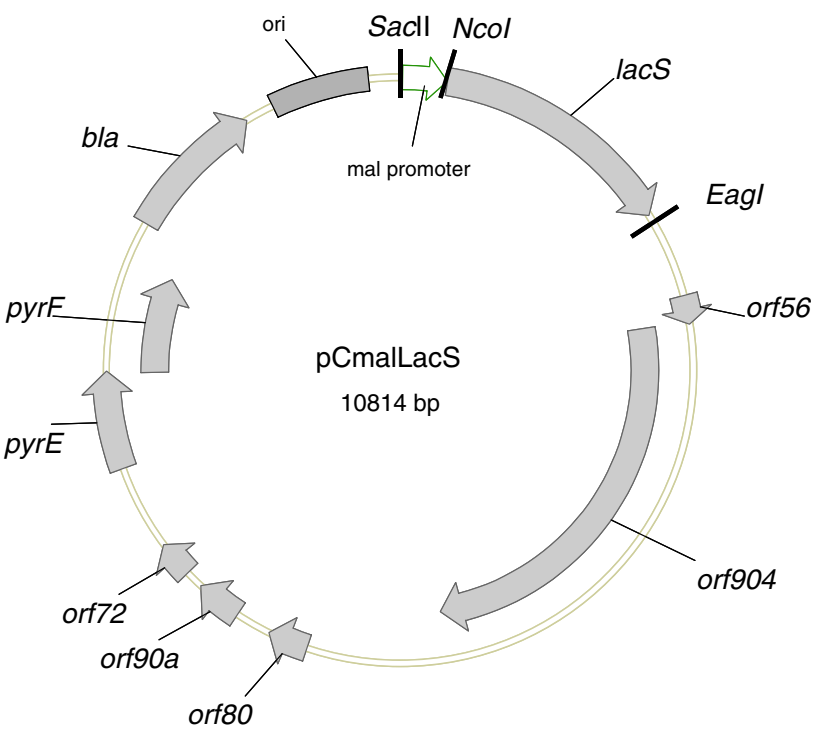

Fig. 1 Vector map of the pRN1-based reporter gene shuttle vector pCmalLacS. lacS reporter gene $\beta$-glycosidase from Sulfolobus solfataricus; orf56, orf904, orf80, orf90a, orf72: genes of the plasmid pRN1; orf56 and orf80 code for DNA-binding proteins and orf904 for a multifunctional replication protein (Lipps, 2009), pyrE (orotate phosphoribosyltransferase) and pyrF (orotidine-5'-phosphate decarboxylases): used as selection marker in Sulfolobus, bla $\beta$-lactamase, ori ColE1 replication origin

amino acids and is strongly bound by streptactin, an engineered streptavidin.

Induction experiments

Single transformants were picked from plates and grown in $50 \mathrm{~mL}$ shake flask cultures in non-inducing medium (either $0.1 \%$ tryptone or $0.1 \%$ tryptone $+0.2 \%$ sucrose). After reinoculation into fresh medium cells were induced by addition of the appropriate sugar(s). Aliquots were withdrawn at the indicated times/ODs, respectively.

\section{$\beta$-galactosidase assay}

Crude extracts were prepared by a freeze-thaw method in which cells were resuspended in $50 \mathrm{mM}$ Na-phosphate buffer, $\mathrm{pH} \mathrm{7}$, and subjected to 5 freeze-thaw cycles (liquid nitrogen $/+50^{\circ} \mathrm{C}$ ). After centrifugation for $30 \mathrm{~min}$ at $10,000 \mathrm{rpm}$ the supernatant was stored at $-20^{\circ} \mathrm{C}$, or assayed directly. All $\beta$-galactosidase assays were conducted in triplicate in a $75^{\circ} \mathrm{C}$ bench top shaker in $1.5 \mathrm{~mL}$ Eppendorf tubes. The reaction mixture consisted of $5 \mu \mathrm{L}$ of crude extract (or water for autohydrolysis control samples), $90 \mu \mathrm{L}$ of $50 \mathrm{mM}$ Na-phosphate buffer, $\mathrm{pH} \mathrm{7,} \mathrm{and} \mathrm{the} \mathrm{assay} \mathrm{was}$ started by addition of $5 \mu \mathrm{L}$ of $12 \mathrm{mg} \mathrm{mL}^{-1}$ ortho-nitrophenyl- $\beta$-D-galactopyranoside (ONPG) solution. Incubation was continued for $2-5$ min depending on the levels of 
Table 2 Overview of the reporter gene constructs used to evaluate the promoter strength and inducibility

sso S. solfataricus $\mathrm{P} 2$, saci $S$. acidocaldarius

${ }^{\text {a }}$ Length from start to end of primer binding sites, last two nucleotides in front of the start codon (ATG or GTG) are replaced by $\mathrm{CC}$ to generate the NcoI site

\begin{tabular}{llll}
\hline Construct name & $\begin{array}{l}\text { Upstream region } \\
\text { of gene }\end{array}$ & $\begin{array}{l}\text { Length of promoter } \\
\text { region }\end{array}$ & Putative inducer \\
\hline pCLacS & - & - & - \\
pCsso_dpsLacS & sso2079 & 152 & $\mathrm{H}_{2} \mathrm{O}_{2}$ \\
pCdpsLacS & saci_1821 & 122 & $\mathrm{H}_{2} \mathrm{O}_{2}$ \\
pCdps_longLacS & saci_1821 & 424 & $\mathrm{H}_{2} \mathrm{O}_{2}$ \\
pClacSLacS & saci_1849 & 641 & Lactose \\
pCmalLacS & saci_1165 & 266 & Maltose, dextrin \\
pCmal2LacS & saci_1165 & 219 & Maltose, dextrin \\
pCmal3LacS & saci_1165 & 169 & Maltose, dextrin \\
pCmal4LacS & saci_1165 & 79 & Maltose, dextrin \\
pCgdhALacS & saci_0155 & 387 & - \\
pCsac7dLacS & saci_0064 & 294 & - \\
\hline
\end{tabular}

activity expected for different samples. Samples known to show high activity (containing the pCsac7dLacS construct) were incubated for a shorter time than samples showing lower activity. This was done to ensure that the orthonitrophenol (NP) production was linear with time over the assay duration as we did an endpoint determination and to make sure to not exceed the linear range for subsequent photometric quantification. At the end of the incubation period the tubes were rapidly cooled on ice and $100 \mu \mathrm{L}$ of $1 \mathrm{M} \mathrm{Na}_{2} \mathrm{CO}_{3}$ solution was added to stop the reaction. For photometric determination of the absorption at $410 \mathrm{~nm}$ the autohydrolysis control samples and the samples (each $200 \mu \mathrm{L}$ ) were transferred from the $1.5 \mathrm{~mL}$ Eppendorf tubes into different wells of a 96-well plate. Additionally, three wells were filled with $200 \mu \mathrm{L}$ of water (blank samples). The measured absorption values at $410 \mathrm{~nm}$ were first corrected for the arithmetic mean of the absorption of the three wells containing only water. The resulting blank corrected absorption values were transformed into NP concentration values by applying a linear calibration function that has been generated with known concentrations of NP and subsequent linear regression to a plot of absorption versus concentration. From the concentration of NP, the mass of NP produced in the autohydrolysis control samples and samples is obtained by multiplying with the volume in the well. Subsequently, the obtained amounts of produced NP in the samples are corrected for the autohydrolysis of the substrate ONPG that cannot be neglected at $75^{\circ} \mathrm{C}$ by subtracting the amount of NP produced in the autohydrolysis controls from the amount of NP produced in the samples. As the calibration function has been derived on mass-based concentrations, the now autohydrolysis corrected amounts of NP are transformed into molar amounts (given in nmol) by dividing by the molar mass of NP. The resulting molar amounts are then divided by the amount (in $\mu \mathrm{g}$ ) of protein contained in the crude extract that has been added to the assay mixture (protein concentration of the crude extracts have been determined by the method of Ehresman (1973). Subsequently the resulting values are divided by the incubation time (in min) to yield the beta-galactosidase activity given in nmol of ONP per $\mu$ g of protein (crude extract) per minute.

\section{Expression of $\mathrm{ABCE} 1$ protein}

Sulfolobus acidocaldarius MR31 was transformed with pSVA1401. A single transformant was picked from the plate and a preculture of $50 \mathrm{~mL}$ was grown to an $\mathrm{OD}_{600}$ of 0.6. $400 \mathrm{~mL}$ of medium either containing no inducer (control) or $0.4 \%$ maltose as inducer was inoculated with $10 \mathrm{~mL}$ of the preculture and grown to an OD of 0.8 . Cell harvest, lysis, ABCE1 protein purification, and detection by Western blot were done as described for SSO0287 in Albers et al. (2006). Briefly, the cells were sonicated and the membranes removed by ultracentrifugation. Then the supernatant was applied to $500 \mu \mathrm{L}$ of HIS-Select material (Sigma). After washing the bound material with 2 column volumes of buffer A $(50 \mathrm{mM}$ HEPES, $100 \mathrm{mM} \mathrm{NaCl}$ and 5 mM Imidazol, pH 8.0), 2 column volumes of buffer B (buffer A with $30 \mathrm{mM}$ Imidazol, $\mathrm{pH}$ 8.0) were applied and then proteins were eluted with buffer $\mathrm{C}$ (buffer A with 250 mM Imidazol, $\mathrm{pH} 7.0$ ).

Xgal test

A qualitative $\beta$-galactosidase assay was based on hydrolysis of 5-bromo-4-chloro-3-indolyl- $\beta$-D-galactoside (X-gal). For liquid cultures $200 \mu \mathrm{L}$ of culture were mixed with $20 \mu \mathrm{L}$ of substrate solution $\left(20 \mathrm{mg} \mathrm{mL}^{-1}\right.$ in dimethylformamide) and incubated at $75^{\circ} \mathrm{C}$ until color development was observed. To score colonies, plates were sprayed with the same $\mathrm{X}$-gal solution and incubated at $75^{\circ} \mathrm{C}$. 
Southern blot

Genomic DNA was prepared from $1 \mathrm{~mL}$ of culture using the Chemagenic DNA Bacteria Kit (Chemagen, Baesweiler, Germany) according to the instructions of the manufacturer. After digestion with XhoI restriction fragments were resolved in $1 \%$ agarose gels, transferred to a Hybond $\mathrm{N}$ membrane (Amersham) by capillary transfer, fixed by UV irradiation for $5 \mathrm{~min}$ on a UV transilluminator, and hybridized to digoxigenin labeled probes complementary to the mal promoter region (266 bp region present in construct pCmalLacS). Labeling and detection were done using the PCR DIG Probe Synthesis Kit and the Digoxigenin Labeling and Detection Kit (Roche).

Copy number determination

Copy number of the construct pCmalLacS was estimated by comparing the band intensity for the fragment corresponding to the linearized pCmalLacS fragment and the fragment containing the genomic copy of the mal promoter region.

\section{Results and discussion}

Identification of inducible promoters and strong promoters

So far only the heat shock promoter tf55 $\alpha$ from $S$. solfataricus had been used with pRN1 shuttle vectors in $S$. acidocaldarius. This construct showed reduced plasmid maintenance in S. acidocaldarius (Berkner et al. 2007). For that reason we tested different promoters to find more suitable ones for expression purposes in S. acidocaldarius.
From literature search (see also Table 3) and own preliminary experiments a number of candidate promoters were chosen for further testing in $S$. acidocaldarius, namely the promoter regions of the $d p s$ genes (coding for a DNA binding protein from starved cells (Maaty et al. 2009; Wiedenheft et al. 2005) from S. solfataricus P2 (sso2079) and $S$. acidocaldarius (saci_1821), of the lacS gene (coding for a $\beta$-glycosidase) from $S$. acidocaldarius (saci_1849), and of the $m b p$ gene (coding for a sugar binding protein, presumably a maltose binding protein) from $S$. acidocaldarius (saci_1165). For strong constitutive expression the promoter region of the $g d h A$ gene (coding for glutamate dehydrogenase, saci_0155) and the sac7d gene (coding for an abundant DNA binding protein, saci_0064) from $S$. acidocaldarius were chosen.

The $d p s$ promoter was selected because the induction of the Dps protein expression under different conditions had already been examined (Wiedenheft et al. 2005), and it had been found that the protein levels increased upon induction with hydrogen peroxide. Furthermore, in microarray experiments a strong induction of the $d p s$ genes of $S$. solfataricus and $S$. acidocaldarius upon UV irradiation has been reported (Gotz et al. 2007).

The lacS promoter from $S$. acidocaldarius was tested because we noticed that $S$. acidocaldarius cells that do not stain blue in an Xgal test on standard growth medium (tryptone/xylose or tryptone/sucrose), do so when cultivated in lactose containing medium. The color development was only very faint; nevertheless, this promoter was studied in more detail.

The $m b p$ promoter (subsequently referred to as mal promoter) was selected after a survey of $A B C$ transport operons for different substrates annotated in the S. acidocaldarius genome. We specifically concentrated on sugarbinding proteins, because induction with sugars does not

Table 3 Transcript abundance and transcript start points

\begin{tabular}{|c|c|c|c|c|c|c|}
\hline \multicolumn{3}{|c|}{ S. acidocaldarius } & \multicolumn{4}{|c|}{ S. solfataricus } \\
\hline Gene & Number & Expression $^{\mathrm{a}}$ & Homologue & Expression $^{\mathrm{a}}$ & Expression $^{\mathrm{b}}$ & Transcript start ${ }^{\mathrm{b}}$ \\
\hline$d p s$ & saci_1821 & 0.4 & sso2079 & 0.3 & 27 & -10 \\
\hline $\operatorname{lac} S^{c}$ & saci_1849 & 0.3 & sso3019 & 0.1 & 7 & $0^{\mathrm{c}}$ \\
\hline $\mathrm{mbp} / \mathrm{mal}$ & saci_1165 & 1.3 & sso1171 & 13.0 & 42 & -14 \\
\hline \multirow[t]{2}{*}{$g d h A$} & saci_0155 & 18.3 & sso1907 & & 36 & -1 \\
\hline & & & sso2044 & 8.9 & 38 & -1 \\
\hline \multirow[t]{2}{*}{$\operatorname{sac} 7 d$} & saci_0064 & & sso10610 & & 159 & -10 \\
\hline & & & sso9535 & & 136 & -7 \\
\hline
\end{tabular}

\footnotetext{
${ }^{a}$ Expression determined by the group of Bernander by microarray experiments. Data are normalized to genomic DNA by hybridizing cDNA versus genomic DNA (Andersson et al. 2006)

b Expression (number of reads/length of transcript) and transcript start points were determined by the group of Sorek on the basis of highthroughput RNA sequencing (Wurtzel et al. 2010)

c In agreement with a study of the group of Paul Blum (Hoang et al. 2004)
} 
negatively influence the cells like it would be the case with hydrogen peroxide for induction with the $d p s$ promoter. Furthermore, sugars are easily available, cheap, and relatively stable under Sulfolobus growth conditions.

The only ABC transport operon (Fig. 5a) predicted to use sugars as substrate in $S$. acidocaldarius consists of the genes saci_1165 (binding protein), saci_1164 and saci_1163 (permease). Saci_1165 is annotated as maltodextrin-binding protein (Chen et al. 2005). Therefore, we tested if the promoter of saci_1165 (mal promoter) was inducible upon maltose addition to a medium containing only tryptone.

The $g d h A$ and the $s a c 7 d$ promoters were chosen based on whole genome expression data (Table 3).

The upstream region of all described genes were fused to the lacS gene in order to measure the promoter strength quantitatively by $\beta$-galactosidase reporter gene assays. The details of the different constructs can be found in Table 2 .

\section{Comparison of different promoter strengths}

S. acidocaldarius MR31 was transformed with the respective plasmids and single transformants were analyzed in $\beta$-galactosidase assays (Fig. 2). No activity could be detected for untransformed S. acidocaldarius MR31 and for the promoterless construct pCLacS. Very low activities were detected for the $d p s$ and the lacS promoter (0.1-0.4 nmol NP per $\mu \mathrm{g}$ of protein per min), low activity for the uninduced mal promoter ( $1 \mathrm{nmol} \mathrm{NP}$ per $\mu \mathrm{g}$ of protein per min), intermediate activity for the induced $\mathrm{mal}$ promoter and the $g d h A$ promoter (10-13 nmol NP per $\mu \mathrm{g}$ of protein per min), and high activity for the $s a c 7 d$ promoter $(45 \mathrm{nmol} \mathrm{NP}$ per $\mu \mathrm{g}$ of protein per min). In general, the activities of the reporter gene assay agree well with the expression data reported by other groups (see Table 3). For the microarray expression data from S. acidocaldarius the Spearman rank correlation is 0.95 , for the RNA sequencing using homologous genes from S. solfataricus the correlation is 0.8 .

The inducibility of the $d p s$ promoter, the lacS promoter, and the mal promoter was initially examined with hydrogen peroxide $(30 \mu \mathrm{M})$, lactose $(0.2-0.4 \%)$, and maltose $(0.2 \%)$, respectively. Whereas for the mal promoter with maltose an apparent increase in promoter activity was visible from Xgal staining of liquid culture aliquots, no changes were observed for the different $d p s$ constructs with $\mathrm{H}_{2} \mathrm{O}_{2}$ (at the same concentration described as inducing by Wiedenheft et al. (2005). First, we attributed this failure to the fact that we used the $d p s$ promoter sequence from $S$. solfataricus (pCssodpsLacS) in the host cells from $S$. acidocaldarius. We therefore cloned the upstream region of the $d p s$ genes from $S$. acidocaldarius (pCdpsLacS), but the resulting construct did not show promoter activity. Then we thought that the upstream region might be too short and increased it to $424 \mathrm{bp}$ (pCdpslongLacS), but still no

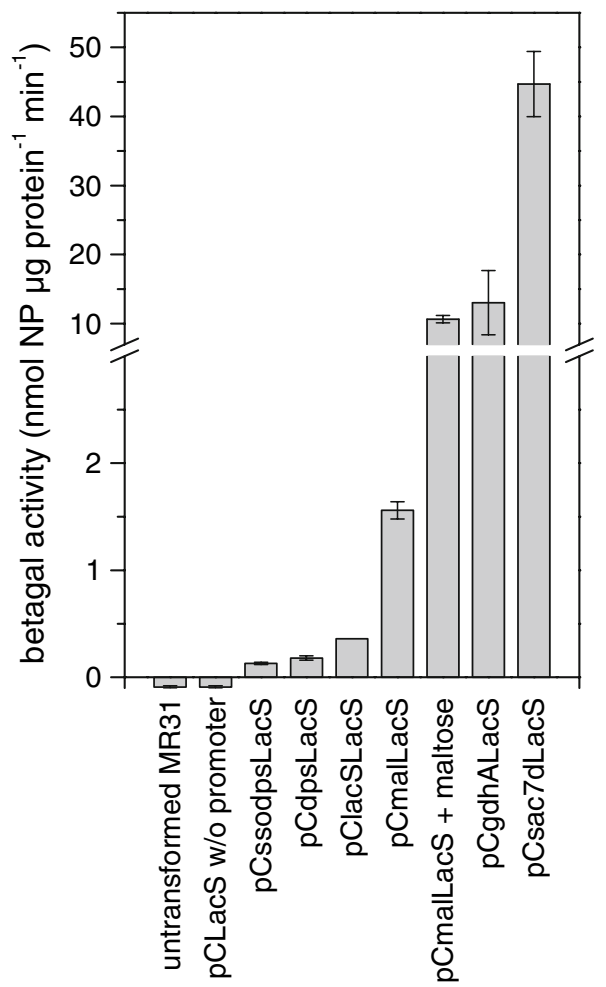

Fig. 2 Comparison of the strength of different promoters in a $\beta$-galactosidase assay (mean of three replicates, error bars correspond to one standard deviation), names of the constructs are explained in Table 2. ODs of the cultures at sampling: MR31: 0.3, pCLacS: 0.4, pCssodpsLacS: 0.3, pCdpsLacS: 0.6, pClacSLacS: 0.6, pCmalLacS (uninduced) 0.4, pCmalLacS + maltose: 0.4 , pCgdhLacS: 0.6 , pCsac7dLacS: 0.2

induction was visible either by Xgal staining or by $\beta$-galactosidase assays. The inducibility of the expression of the Dps protein with $\mathrm{H}_{2} \mathrm{O}_{2}$ has clearly been demonstrated (Wiedenheft et al. 2005). However, the induction of the protein production might be regulated by a mechanism other than transcriptional regulation or the necessary operator regions might be found within the $d p s$ gene itself, which is in the reporter gene constructs replaced by the $l a c S$ gene. It is also possible that iron concentration in the media used for cultivation might influence $d p s$ expression. In that regard, the highly variable expression obtained in different microarray experiments should also be considered (see Table 3). For the lacS promoter a minimal increase in $\beta$-galactosidase reporter activity was observed, but the resulting activity was still very weak. Therefore, the mal promoter seemed to be the most suitable candidate for further characterization.

Inducibility of the mal promoter

To further examine the kinetics of the mal promoterdirected expression during a batch culture, samples from cultures containing pCmalLacS were analyzed in early log 
phase, late log phase, and stationary phase (Fig. 3a) from uninduced cultures and cultures induced by either 0.2 or $0.4 \%$ maltose or dextrin, respectively (Fig. $3 b$ ).

The promoter has low basal activity and is inducible upon addition of maltose and dextrin up to 17-fold (Fig. 3b). Induction is already visible in early log phase and increases to late log phase before declining in stationary phase. Only for dextrin at $0.4 \%$ a further increase in stationary phase is visible. It should be kept in mind that this could be a secondary effect due to the high nutrient concentration present in this medium. In late log phase (Fig. 3b) induction is comparable for dextrin and maltose. Although it is very probable that dextrin is broken down to maltose under the conditions of the Sulfolobus growth medium, it has been noted earlier that dextrin is an
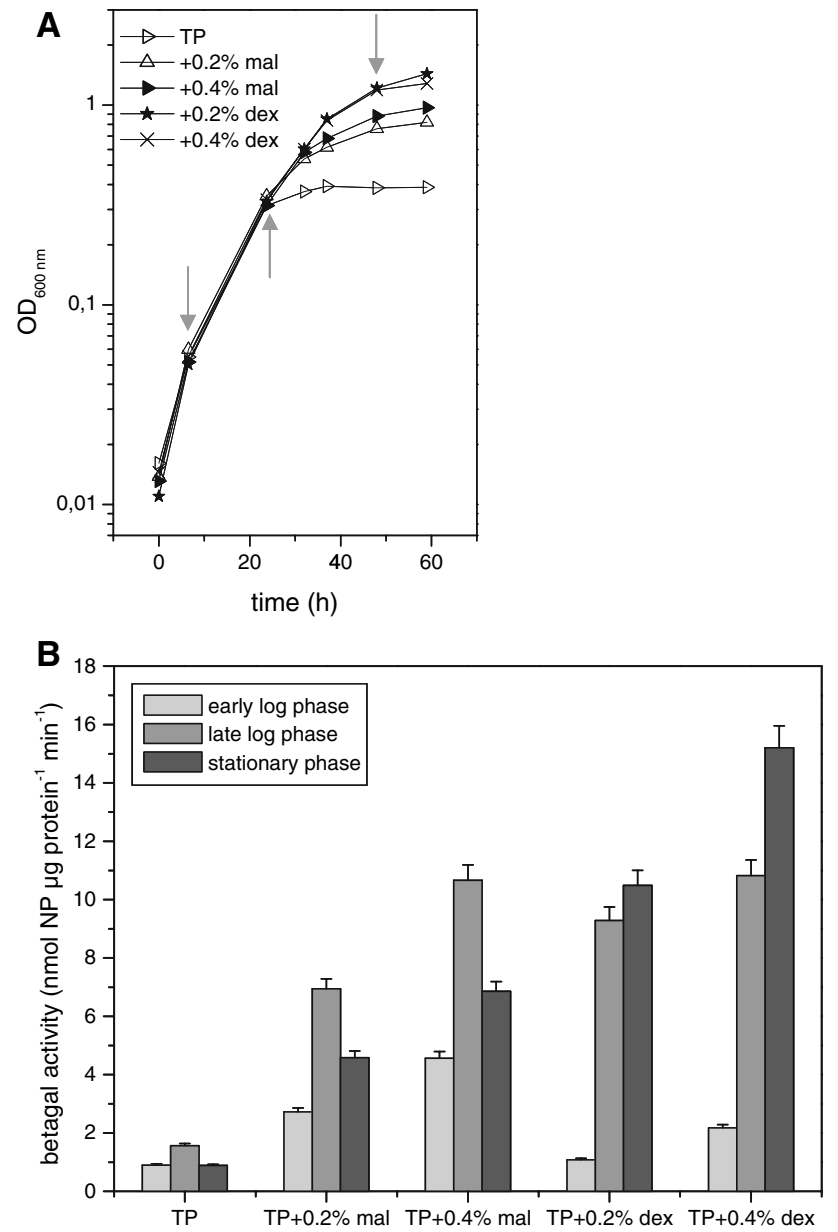

Fig. 3 Expression of the $\beta$-galactosidase under the control of the mal promoter. a Growth curves of five different cultures obtained by 1:99 dilution of a preculture into the respective growth media, TP uninduced culture, + mal addition of the indicated amount of maltose, $+d e x$ addition of the indicated amount of dextrin at time point 0 . The sampling times are indicated by gray arrows. b Measured $\beta$-galactosidase activities for the indicated sampling time points in A [early log phase (first arrow), late log phase (second arrow), stationary phase (third arrow) from left to right] especially suitable substrate for the growth of $S$. acidocaldarius (Grogan 1989). We cannot rule out that the induction effect is not alone due to dextrin itself but could also be attributed to some breakdown products of dextrin other than maltose that may also act as inducers on the mal promoter, e.g., maltotriose.

\section{Influence of different sugars}

Next we tested the influence of different sugars on the basal activity and the inducibility of the mal promoter. Besides maltose and dextrin, glucose, sucrose, and xylose were tested alone and in combination with maltose (Fig. 4).

With glucose, dextrin, and xylose higher basal activity levels were observed than with tryptone alone. Only sucrose did not lead to higher basal activity. It should be noted that the medium tryptone/sucrose supports a growth comparable to tryptone/maltose (data not shown). The induction level compared with the tryptone-tryptone/ maltose cultures were lower for glucose, dextrin, and xylose, but comparable for sucrose. The addition of another sugar besides the one used for induction is beneficial for the cell growth, as can be seen from Fig. 3a. In tryptone only containing medium the stationary phase is reached early and the final turbidity of the cultures are considerably lower than for the media containing additional sugars. Thus, these experiments suggest that a tryptone/sucrose medium is most suitable for growth without induction

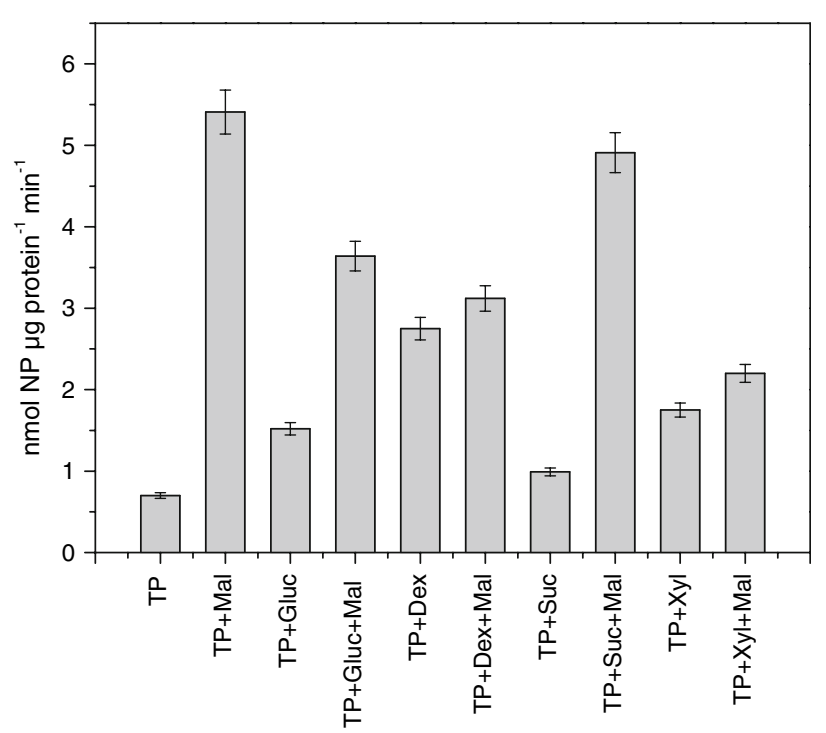

Fig. 4 Influence of different sugars on the basal activity and the inducibility of the mal promoter in $\beta$-galactosidase reporter gene assays (mean $\pm \mathrm{SD}$ ). TP tryptone, Mal maltose, Gluc glucose, Dex dextrin, Suc sucrose, $X y l$ xylose. All sugars were added at $0.2 \%$ final concentration. ODs at sampling: TP: $0.3, \mathrm{TP}+\mathrm{Mal}: 0.2, \mathrm{TP}+$ Gluc: 0.2, TP + Gluc + Mal: 0.2, TP + Dex: 0.2, TP + Dex + Mal: 0.2, $\mathrm{TP}+$ Suc: $0.4, \mathrm{TP}+$ Suc + Mal: 0.3, TP + Xyl: 0.5, TP + Xyl + Mal: 0.5 
followed by addition of maltose for induction of the mal promoter. As the reporter gene activity reaches its maximum after $30-50 \mathrm{~h}$, the inducer should be added shortly after or concurrent with the inoculation of a culture for protein expression purposes.

For some applications of the mal promoter repression of the observed basal activity of the mal promoter would be desirable. For $S$. solfataricus the expression of $\alpha$-glycosidase, $\beta$-glycosidase, and $\alpha$-amylase has been shown to be repressed by certain sugars and amino acids (Haseltine et al. 1996, 1999, Hoang et al. 2004). Furthermore, the expression of the arabinose transport operon is also regulated differently in the presence of amino acids in the growth medium (Lubelska et al. 2006). From our experiments it became clear that none of the sugars tested had a repressing effect on the basal activity of the mal promoter. For that reason we further tested the addition of glutamine or a mixture of all 20 amino acids (at final concentrations of 0.5 and $0.1 \%$, respectively) for their ability to repress the mal promoter. However, none of the tested substances showed a clear repressing effect on the basal promoter activity in reporter gene experiments (data not shown).

\section{Determination of the minimal promoter region}

To narrow down the upstream region that is necessary for promoter function, i.e., inducibility by maltose, we stepwise deleted the upstream region (Fig. 5). Whereas constructs comprising 266-169 bp of upstream region were still inducible by maltose, a shorter construct comprising only $79 \mathrm{bp}$ showed only basal activity after addition of maltose. Therefore, the sequence part responsive to maltose is situated within 169 bp upstream of the ATG start codon. When comparing the promoter strength of the inducible variants, no changes in inducibility were visible for the three constructs within the observed variations for parallel experiments. Therefore, the constructs pCmalLacS (266 bp), pCmal2LacS (219 bp), and pCmal3LacS (169 bp) can therefore, in principle, be used interchangeably for expression purposes.

Plasmid maintenance and copy number

of the expression vector pCmalLacS

For the routine use of a shuttle vector for expression purposes a high retention of the construct in the host cell is highly desirable. As already described for the shuttle vector pJlacS in comparison with $\mathrm{pJ}$ without expression cassette (Berkner et al. 2007), the additional sequences to the shuttle vector with high retention may alter this behavior. Therefore, we tested the plasmid retention of the expression vector pCmalLacS under selective and non-selective conditions. After 200 generations in selective liquid medium
A

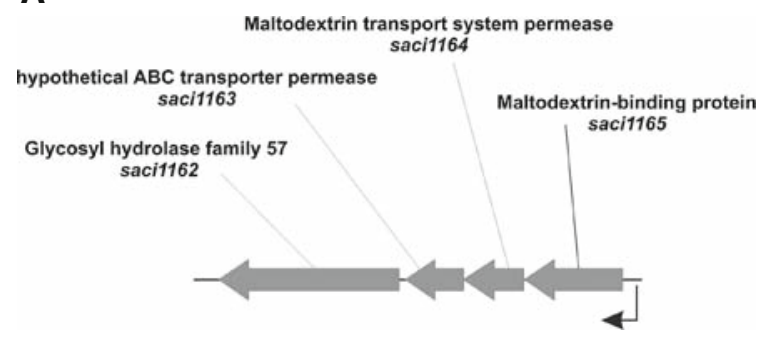

B

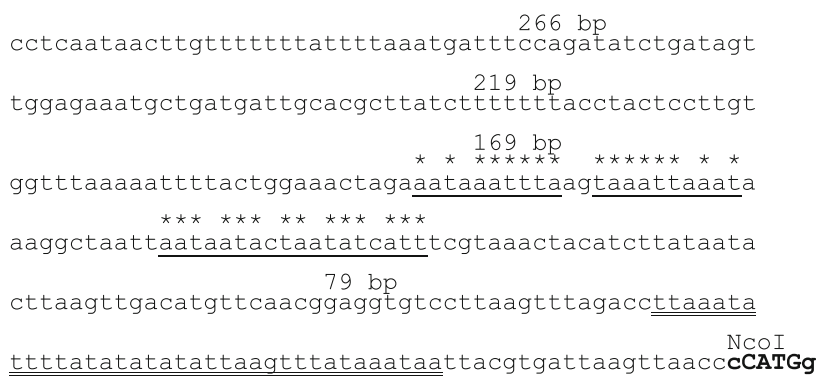

C

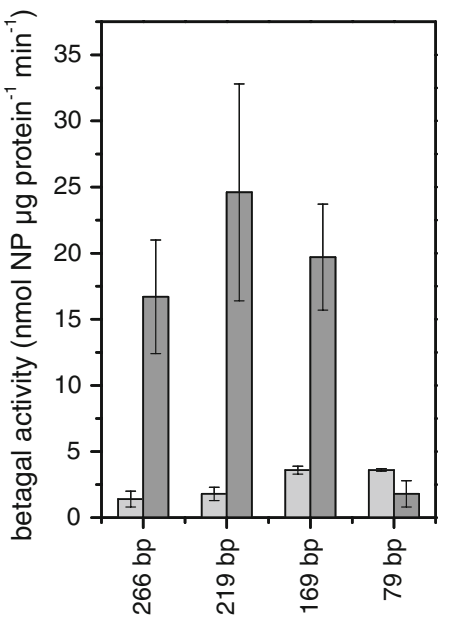

Fig. 5 Determination of the minimal sequence region necessary for inducibility of the mal promoter. a Operon structure, b promoter sequence (the start codon and the engineered NcoI restriction site is in bold). The beginning of the promoter region of each deletion construct is indicated with the number of base pairs remaining above the sequence. Two putative regulatory imperfect palindromic binding sites (18-20 bases) are underlined and the matching bases have an asterisk above the sequence. The region of the predicted BRE-TATA box is double underlined. c $\beta$-galactosidase measurements of the deletion constructs (mean \pm SD). The activities obtained in the absence of maltose are shown in light bars and the activities in the presence of maltose in dark bars. ODs at sampling: pCmal1LacS both $-/+$ maltose 0.1 , all other constructs: 0.2

(tryptone/sucrose), all cells still contained a shuttle vector as shown by plating and Xgal staining of the colonies. All colonies were found to stain blue (data not shown). The vector loss under non-selective conditions was also monitored by plating aliquots of a culture incubated with uracil on selective and non-selective plates and determining the 
fraction of shuttle vector containing cells. After four generations in non-selective medium still $95 \%$ of all cells contained a shuttle vector and after seven generations the vector was lost to approximately $50 \%$ (data not shown). These experiments show that the vector pCmalLacS has a high retention under selective conditions and is only slowly lost under non-selective conditions. We would like to point out that this behavior is not found for all of our tested promoter constructs. Especially the constructs with very strong promoter activity (e.g., pCsac7dLacS) show decreased plasmid retention and develop only small colonies on plates. The use of these promoters is nevertheless possible, but cultures have always to be kept under stringent selection conditions because otherwise the vector is rapidly lost. This also implies that cultures should not reach stationary phase as the selective pressure from uracil selection necessary for the maintenance of shuttle vectors decreases when a higher number of cells die due to nutrient limitations and uracil is set free from the degrading cells. It should also be kept in mind that the plasmid maintenance of the vector is influenced by the gene cloned behind the promoter for expression purposes. In case the expressed product has a negative impact on cell fitness the vector might be lost more rapidly.

We also determined the copy number of the vector pCmalLacS by Southern blotting (Fig. 6) and found it to be around 10 per chromosome at an OD of 0.2 and around 5 per chromosome at an OD of 0.9. The copy number seems to follow the same pattern as has been observed for the vector pC (Berkner et al. 2007): low copy numbers when the culture is inoculated, then an increase of the copy number with a maximum at approximately OD 0.2 to OD 0.6 , and a subsequent decline of the copy number at higher

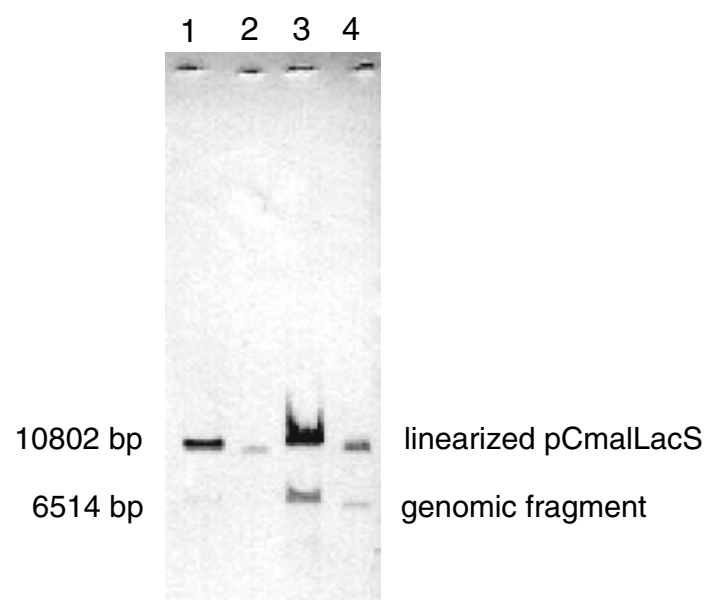

Fig. 6 Southern blot for copy number determination of construct pCmalLacS in S. acidocaldarius MR31. The culture shown in lane 1 and 2 was sampled at OD of 0.2, the culture shown in lane 3 and 4 was sampled at an OD of 0.9. In lane 2 and 4 1/9th of the amount in lane 1 and 3 has been loaded onto the gel
ODs. Therefore, it should be noted that the measured expression levels are likely to show a copy number effect. This might be seen in the data presented in Fig. 3b. The uninduced tryptone only containing culture shows an increase in activity in late log phase compared with early $\log$ phase and stationary phase. This effect is likely to occur in the induced cultures, too.

Protein expression with the mal promoter

The ABCE1 protein is an evolutionary highly conserved protein that is thought to be involved in recognition and modification of RNA assemblies (Barthelme et al. 2007). The 68-kDa protein ABCE1 (SSO0287) from S. solfataricus was expressed as a C-terminal $\mathrm{His}_{10^{-}}$and strep-tag fusion protein. ABCE1 was purified by His-Select $\mathrm{Ni}$ affinity chromatography, yielding $1 \mathrm{mg}$ purified enzyme per L of cells (Fig. 7). Immunodetection with His tagspecific antibodies and Streptactin confirmed that the purified protein corresponded to the recombinant tagged protein. When comparing the protein yield from the uninduced and induced culture it became obvious that the mal promoter shows basal activity, as also visible from the reporter gene experiments, because also in the uninduced culture ABCE1 protein is produced in considerable amounts (data not shown). However, an approximately fivefold increase in protein production after induction with maltose was observed.

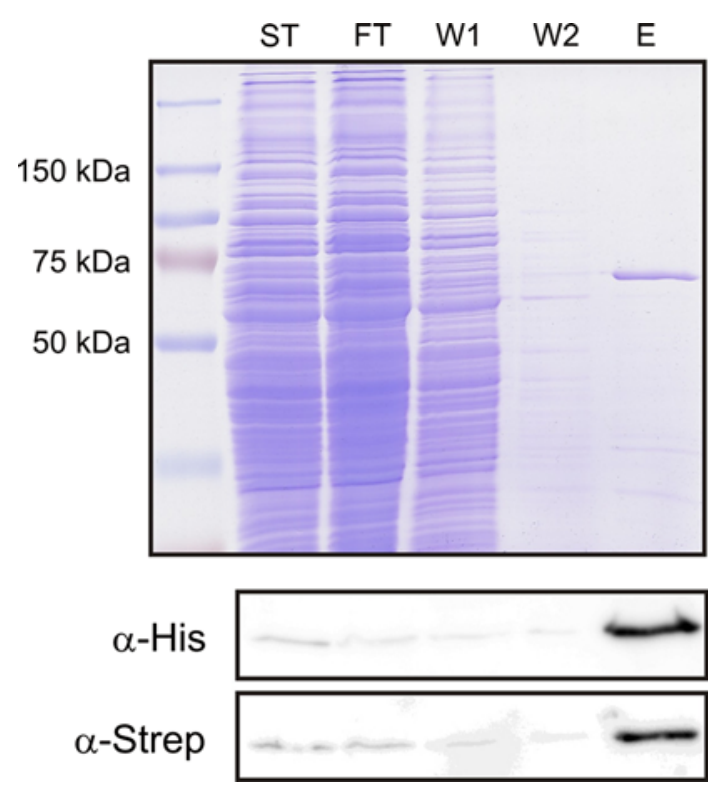

Fig. 7 Purification of SSO0287 expressed in S. acidocaldarius by affinity chromatography. The upper panel shows the Coomassie stained gel of the different fractions of the purification procedure. The same samples were used for Western blotting using both, anti-Histag and Streptactin antibodies. ST starting material, FT flow through, WI and $W 2$ wash steps, $E$ elution 


\section{Conclusions}

In establishing $S$. acidocaldarius as an emerging model organism the development of genetic tools is of essential importance. The promoter of the maltose-binding protein (Saci_1165) has been identified as a suitable inducible promoter for controlled expression using the pRN1-based shuttle vector $\mathrm{pC}$ in $S$. acidocaldarius. The promoter shows low basal activity and an increase in expression up to 17 -fold upon addition of $0.2-0.4 \%$ maltose or dextrin in reporter gene experiments. For the expressed ABCE1 protein an increase in expression of about fivefold was observed. As promoters with only very low activity we identified the $d p s$ promoter and the lacS promoter from $S$. acidocaldarius. Promoters that show high to very high constitutive expression are the $g d h A$ and the $s a c 7 d$ promoter.

For routine use the $\mathrm{mal}$ promoter is recommended due to its stable plasmid maintenance and its successful use for protein expression in S. acidocaldarius.

Acknowledgments The work of G.L. has been supported by the DFG (Li913-6). S.V.A. and A.W. were supported by a VIDI grant of the Dutch Science Organization (NWO) and by intramural funds of the Max Planck Society.

Open Access This article is distributed under the terms of the Creative Commons Attribution Noncommercial License which permits any noncommercial use, distribution, and reproduction in any medium, provided the original author(s) and source are credited.

\section{References}

Albers SV, Koning SM, Konings WN, Driessen AJ (2004) Insights into $\mathrm{ABC}$ transport in archaea. J Bioenerg Biomembr 36:5-15

Albers SV, Jonuscheit M, Dinkelaker S, Urich T, Kletzin A, Tampe R, Driessen AJ, Schleper C (2006) Production of recombinant and tagged proteins in the hyperthermophilic archaeon Sulfolobus solfataricus. Appl Environ Microbiol 72:102-111

Andersson AF, Lundgren M, Eriksson S, Rosenlund M, Bernander R, Nilsson P (2006) Global analysis of mRNA stability in the archaeon Sulfolobus. Genome Biol 7:R99

Barthelme D, Scheele U, Dinkelaker S, Janoschka A, Macmillan F, Albers SV, Driessen AJ, Stagni MS, Bill E, Meyer-Klaucke W, Schunemann V, Tampe R (2007) Structural organization of essential iron-sulfur clusters in the evolutionarily highly conserved ATP-binding cassette protein ABCE1. J Biol Chem 282:14598-14607

Berkner S, Lipps G (2008a) Genetic tools for Sulfolobus spp.: vectors and first applications. Arch Microbiol 190:217-230

Berkner S, Lipps G (2008b) Mutation and reversion frequencies of different Sulfolobus species and strains. Extremophiles 12:263-270

Berkner S, Grogan D, Albers SV, Lipps G (2007) Small multicopy, non-integrative shuttle vectors based on the plasmid pRN1 for Sulfolobus acidocaldarius and Sulfolobus solfataricus, model organisms of the (cren-)archaea. Nucleic Acids Res 35:e88

Brock TD, Brock KM, Belly RT, Weiss RL (1972) Sulfolobus: a new genus of sulfur-oxidizing bacteria living at low $\mathrm{pH}$ and high temperature. Arch Mikrobiol 84:54-68
Chen L, Brugger K, Skovgaard M, Redder P, She Q, Torarinsson E, Greve B, Awayez M, Zibat A, Klenk HP, Garrett RA (2005) The genome of Sulfolobus acidocaldarius, a model organism of the Crenarchaeota. J Bacteriol 187:4992-4999

Deng L, Zhu H, Chen Z, Liang YX, She Q (2009) Unmarked gene deletion and host-vector system for the hyperthermophilic crenarchaeon Sulfolobus islandicus. Extremophiles 13:735-746

Duggin IG, McCallum SA, Bell SD (2008) Chromosome replication dynamics in the archaeon Sulfolobus acidocaldarius. Proc Natl Acad Sci USA 105:16737-16742

Ehresmann B, Imbault P, Weil JH (1973) Spectrophotometric determination of protein concentration in cell extracts containing tRNA's and rRNA's. Anal Biochem 54:454-463

Elferink MG, Albers SV, Konings WN, Driessen AJ (2001) Sugar transport in Sulfolobus solfataricus is mediated by two families of binding protein-dependent $\mathrm{ABC}$ transporters. Mol Microbiol 39:1494-1503

Gotz D, Paytubi S, Munro S, Lundgren M, Bernander R, White MF (2007) Responses of hyperthermophilic crenarchaea to UV irradiation. Genome Biol 8:R220

Grogan DW (1989) Phenotypic characterization of the archaebacterial genus Sulfolobus: comparison of five wild-type strains. J Bacteriol 171:6710-6719

Haseltine C, Rolfsmeier M, Blum P (1996) The glucose effect and regulation of alpha-amylase synthesis in the hyperthermophilic archaeon Sulfolobus solfataricus. J Bacteriol 178:945-950

Haseltine C, Montalvo RR, Bini E, Carl A, Blum P (1999) Coordinate transcriptional control in the hyperthermophilic archaeon Sulfolobus solfataricus. J Bacteriol 181:3920-3927

Hoang V, Bini E, Dixit V, Drozda M, Blum P (2004) The role of cisacting sequences governing catabolite repression control of lacS expression in the archaeon Sulfolobus solfataricus. Genetics 167:1563-1572

Jonuscheit M, Martusewitsch E, Stedman KM, Schleper C (2003) A reporter gene system for the hyperthermophilic archaeon Sulfolobus solfataricus based on a selectable and integrative shuttle vector. Mol Microbiol 48:1241-1252

Kurosawa N, Grogan DW (2005) Homologous recombination of exogenous DNA with the Sulfolobus acidocaldarius genome: properties and uses. FEMS Microbiol Lett 253:141-149

Lipps G (2009) Molecular biology of the pRN1 plasmid from Sulfolobus islandicus. Biochem Soc Trans 37:42-45

Lubelska JM, Jonuscheit M, Schleper C, Albers SV, Driessen AJ (2006) Regulation of expression of the arabinose and glucose transporter genes in the thermophilic archaeon Sulfolobus solfataricus. Extremophiles 10:383-391

Maaty WS, Wiedenheft B, Tarlykov P, Schaff N, Heinemann J, Robison-Cox J, Valenzuela J, Dougherty A, Blum P, Lawrence CM, Douglas T, Young MJ, Bothner B (2009) Something old, something new, something borrowed; how the thermoacidophilic archaeon Sulfolobus solfataricus responds to oxidative stress. PLoS One 4:e6964

Reilly MS, Grogan DW (2001) Characterization of intragenic recombination in a hyperthermophilic archaeon via conjugational DNA exchange. J Bacteriol 183:2943-2946

Samson RY, Obita T, Freund SM, Williams RL, Bell SD (2008) A role for the ESCRT system in cell division in archaea. Science 322:1710-1713

She Q, Zhang C, Deng L, Peng N, Chen Z, Liang YX (2009) Genetic analyses in the hyperthermophilic archaeon Sulfolobus islandicus. Biochem Soc Trans 37:92-96

Wagner M, Berkner S, Ajon M, Driessen AJ, Lipps G, Albers SV (2009) Expanding and understanding the genetic toolbox of the hyperthermophilic genus Sulfolobus. Biochem Soc Trans 37:97-101 
Wiedenheft B, Mosolf J, Willits D, Yeager M, Dryden KA, Young M, Douglas T (2005) From the cover: an archaeal antioxidant: characterization of a Dps-like protein from Sulfolobus solfataricus. Proc Natl Acad Sci USA 102:10551-10556
Wurtzel O, Sapra R, Chen F, Zhu Y, Simmons BA, Sorek R (2010) A single-base resolution map of an archaeal transcriptome. Genome Res 20:133-141 